\title{
Micromachined polycrystalline thin film temperature sensors
}

\author{
Linan Jiang $\dagger$, Man Wong $\ddagger$ and Yitshak Zohar $\dagger$ \\ $\dagger$ Department of Mechanical Engineering, Hong Kong University of Science and Technology, \\ Clear Water Bay, Kowloon, Hong Kong, People's Republic of China \\ \$ Department of Electrical \& Electronic Engineering, Hong Kong University of Science and \\ Technology, Clear Water Bay, Kowloon, Hong Kong, People's Republic of China
}

Received 17 December 1997, in final form and accepted for publication 29 July 1998

\begin{abstract}
Polycrystalline thin films based on two elemental semiconducting materials, $\mathrm{Si}$ and $\mathrm{Ge}$, have been utilized to fabricate microthermistors. The thermistors are designed in a heavy-light-heavy doping concentration arrangement. The design, fabrication, analysis and characterization of a variety of thermistors under different doping schemes is described. Finally, the operation of the thermistors in self-heating operation is discussed. The results provide a systematic framework for the application of semiconducting microthermoresistors.
\end{abstract}

Keywords: temperature microsensors, polycrystalline semiconductors, thermoresistors, integrated microsystems

\section{Introduction}

Thermal sensors are used to measure various heat-related quantities such as temperature, heat flux, heat capacity, etc. Among these quantities, temperature is perhaps the most fundamental one. For example, the amount of heat in a body is a product of its absolute temperature and its heat capacity. The latter characterizes the capability of a given material to store heat energy. Because temperature is such an important process parameter, it must be monitored and controlled. Thus, it is not surprising that thermal sensors form the largest and perhaps the most important class of microsensors today. About $40 \%$ of all solid-state sensors commercially sold are thermal sensors (Gardner 1994).

Thermal sensors can be classified either as contacting or non-contacting. Contacting sensors physically touch the heat source or sink of interest. The thermal 'signal' then propagates from the source or sink to the sensing element, which in response either generates or modulates an electrical signal. On the other hand, non-contacting thermal sensors sense heat remotely, without physically touching the source or sink. While a few thermal-electrical sensors are self-generating, such as a thermocouple generating a temperature-dependent electromotive force, the majority is modulating. Many of the thermoconductive sensors, such as thermoresistors, thermodiodes, or thermotransistors etc, can be made as microsensors and used to measure a wide range of temperatures from as low as $1.5 \mathrm{~K}$ to as high as $2700 \mathrm{~K}$.

The working principle of thermistors is based on the temperature dependence of the electrical resistivity of the materials. Although the resistivity of both metals and semiconductors vary with temperature, their behaviour can be quite different. In metals, the resistivity change is essentially caused by changes in carrier mobility, which typically decreases with temperature because of enhanced phonon scattering. On the other hand, greater sensitivity can be obtained in highly resistant singlecrystalline semiconducting materials, in which the free carrier concentration (resistivity) increases (decreases) exponentially with temperature. While the use of intrinsic (undoped) semiconductors for thermistors is attractive because of the high-temperature sensitivity, parameter control and device stability usually suffer. Therefore, it is more practical to use extrinsic (doped) semiconductors, which become quasimetallic at a sufficiently high doping level (Gardner 1994).

More popularly, thermistors built on polycrystalline semiconducting thin films are used. Such devices exhibit even higher temperature sensitivity than those realized on extrinsic single-crystalline materials because of conduction across grain boundaries. Matrangelo (1990) presented a detailed study of a uniformly doped bridge. Tai et al (1988) studied a similar bridge but with the centre part being lightly doped. Recently, Jiang (1997) applied the bridge design to construct a thermal shear-stress sensor. In this paper, we describe the fabrication, the analyses and the characterization of temperature microsensors based on polycrystalline thin films of two elemental semiconducting materials- $\mathrm{Si}, \mathrm{Ge}-$ and their alloys. Non-uniform doping of boron and phosphorus are investigated in a bridge-like arrangement. The emphasis is on developing a process which is fully compatible with current integrated circuits (IC) technology to allow the integration of micromechanical elements with microelectronic devices on a single chip. The microsystem for surface shear-stress reduction (Ho et al 1997) or the microsystem for cooling of microdevices (Jiang et al 1998) are examples for these applications. 


\section{Polycrystalline thermistors}

Conduction in polycrystalline silicon (or poly-Si for short) films has been extensively studied since they became technologically important more than 20 years ago. It has been recognized that a proper model for the analysis of polycrystalline materials should take into account the existence of multiple crystallite grains in the films (Lu et al 1980). The resistivity, $\chi$, of poly-Si is modelled to arise from a series combination of two terms: 'bulk' resistivity within a grain, $\chi_{c}$, which is controlled by regular phonon and impurity scattering processes, and 'barrier' resistivity across the grain boundaries, $\chi_{b}$, which is controlled by a thermionic emission process. This resistivity model can be easily extended to polycrystalline SiGe alloy thermistors by suitably adjusting the grain boundary trap density, the carrier mobility inside the neutral grain body and the band-gap energy. It turns out that $\chi_{b}$ dominates from light to moderate doping. The resistivity is highly sensitive to light doping concentration in the range between $10^{16}$ and $10^{18} \mathrm{~cm}^{-3}$, which makes it difficult to accurately control film resistivity. Therefore, in this work, moderate doping concentrations between $10^{18}$ and mid- $10^{19} \mathrm{~cm}^{-3}$ have been selected for more stable parameter control. For high doping, above $10^{20} \mathrm{~cm}^{-3}, \chi_{3}$ dominates and the resistivity becomes relatively insensitive to doping concentration variations.

A hyperbolic sine dependence of the current, $I$, on the applied voltage, $V$, can be derived for a conduction mechanism controlled by thermionic emission (Jorsh and Muller 1978):

$$
I=I_{0} \sinh \frac{V}{V_{0}} \approx I_{0} \frac{V}{V_{0}} \Rightarrow R \equiv \frac{V}{I} \approx \frac{V_{0}}{I_{0}}
$$

where the approximations apply when the voltage drop across the grain boundaries is small compared with $(k T / q)$. The parameters $I_{0}$ and $V_{0}$ are given by:

$$
I_{0}=2 A A^{*} T^{2} \mathrm{e}^{-\frac{q V_{B 0}}{k T}}
$$

and

$$
V_{0}=\frac{2 k T N}{q}
$$

where $T$ is the temperature of the thermistor, $A$ is the crosssectional area perpendicular to the thermistor current flow, $A^{*}$ is the Richardson constant, $V_{B 0}$ is the barrier height at each grain boundary, $N$ is the number of grains in the thermistor, $q$ is the electron charge and $k$ is Boltzmann's constant. Once the resistance, $R$, is known, the resistivity can be calculated as,

$$
\chi=\frac{R \cdot A}{L}
$$

where $L$ is the length of the resistor. If it is assumed that, to a large extent, the conduction in moderately doped polycrystalline thermistor is still dominated by thermionic emission, then the following expression for the temperature coefficient of resistance (TCR) can be obtained:

$$
\lambda=\frac{\mathrm{d} \ln R}{\mathrm{~d} T}=-\frac{1}{T}-\frac{q V_{B 0}}{k T^{2}}
$$

where $\lambda$, denoting the TCR, is negative and nonlinear in $T$. For moderately doped polycrystalline materials, the grains are only partially depleted and $V_{B 0}$ can be approximated by:

$$
V_{B 0} \approx \frac{Q_{T}^{2}}{8 q \varepsilon N_{d}}
$$

where $Q_{T}$ is the trap charge per unit grain boundary area, $\varepsilon$ is the dielectric constant, and $N_{d}$ is the doping concentration. Clearly $V_{B 0}$, hence also $|\lambda|$, decreases with doping concentration.

The resistivity of different films as a function of the doping concentration is shown in figure 1. Figure 1(a) compares the calculated resistivity for poly-Si film, where the average grain size is about $300 \AA$ (Lu et al 1980) with experimental measurements of the resistivity of various boron-doped films. The calculations are based on equation (4). For moderate doping concentration, $10^{18}$ to mid- $10^{19} \mathrm{~cm}^{-3}$, the resistivity of the poly-Si decreases exponentially, consistent with previous data (Obermer et al 1986). However, the resistivity of poly-Ge and poly-SiGe decreases almost linearly with the doping concentration at this range. At the lower doping concentration, the resistivity of the poly-Si is clearly larger than both poly-SiGe and poly$\mathrm{Ge}$. At the higher doping concentration, the resistivity of all three materials is about the same. Similarly, figure 1(b) shows that the resistivity of poly-Si and poly-SiGe decreases as a function of phosphorus doping concentration. However, here at doping concentrations of about $10^{19} \mathrm{~cm}^{-3}$, the resistivity of the poly-SiGe is slightly higher than that of the poly-Si.

The relative resistance change of boron-doped and phosphorus-doped polycrystalline films due to temperature change is shown in figures $2(a)$ and $(b)$, respectively, with the doping concentration as the varying parameter. At moderate doping levels, $1.25 \times 10^{18}$ to $1.25 \times 10^{19} \mathrm{~cm}^{3}$, the behaviour is like undoped semiconductors, where the thermionic emission mechanism is dominant and the temperature dependence is exponential with negative TCR. At a higher doping level, $10^{20} \mathrm{~cm}^{3}$, the behaviour is like metals, where the carrier mobility mechanism is dominant and the temperature dependence is nearly linear with positive TCR. In general, the temperature dependence increases with decreasing doping concentration. The dependence of the TCR on doping concentration is shown in figure 3. For boron-doped film, $\lambda$ approaches zero or a slightly positive value as the concentration increases from $1.25 \times 10^{18}$ to $1.25 \times 10^{19} \mathrm{~cm}^{3}$. The calculated TCR for poly-Si film based on equation (5) is in close agreement with the experimental data, and its dependence on the doping concentration is highly nonlinear. The largest negative TCR value measured for boron-doped poly-Si film with concentration of $1.25 \times 10^{18} \mathrm{~cm}^{3}$ is about $-0.03{ }^{\circ} \mathrm{C}$.

\section{Sensor design and fabrication}

The performance requirements of a sensor dictate its design. Two of the most important requirements are sensitivity and stability. It is well known that lightly doped films, while less stable, are much more sensitive than highly doped films which are more stable. In order to satisfy these two conflicting 

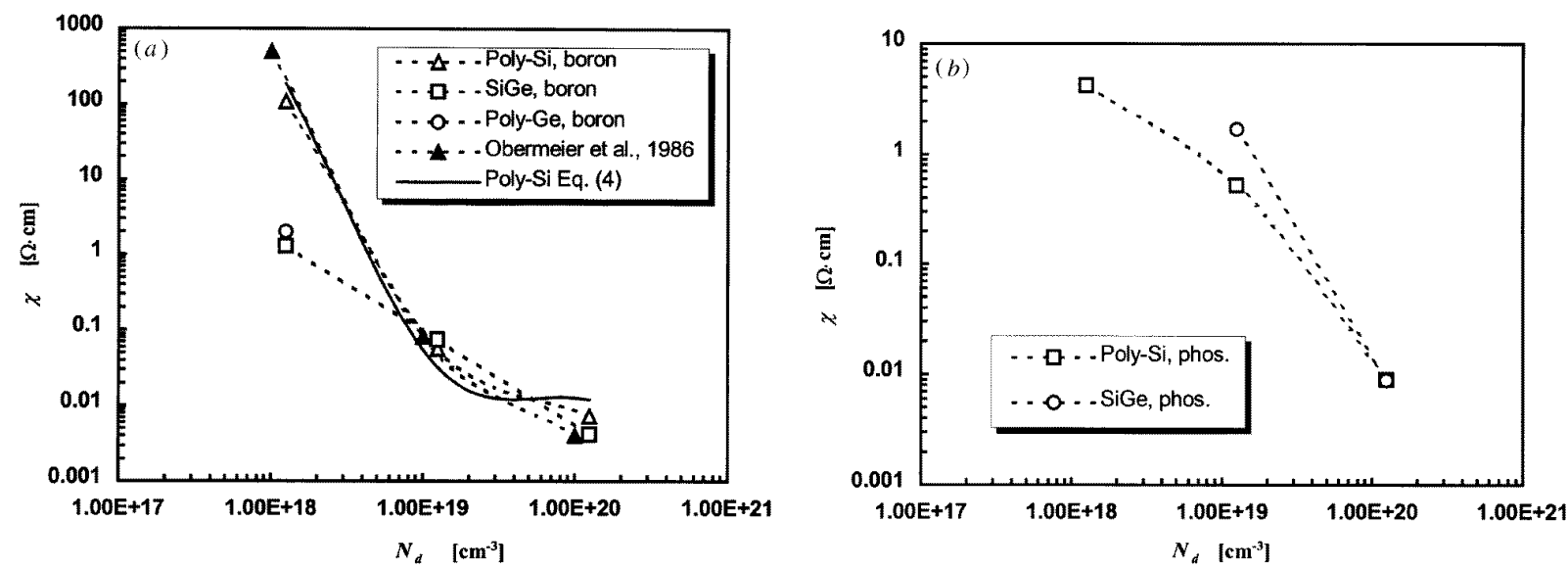

Figure 1. Dependence of resistivity on doping concentration: $(a)$ boron doped, and $(b)$ phosphorus doped
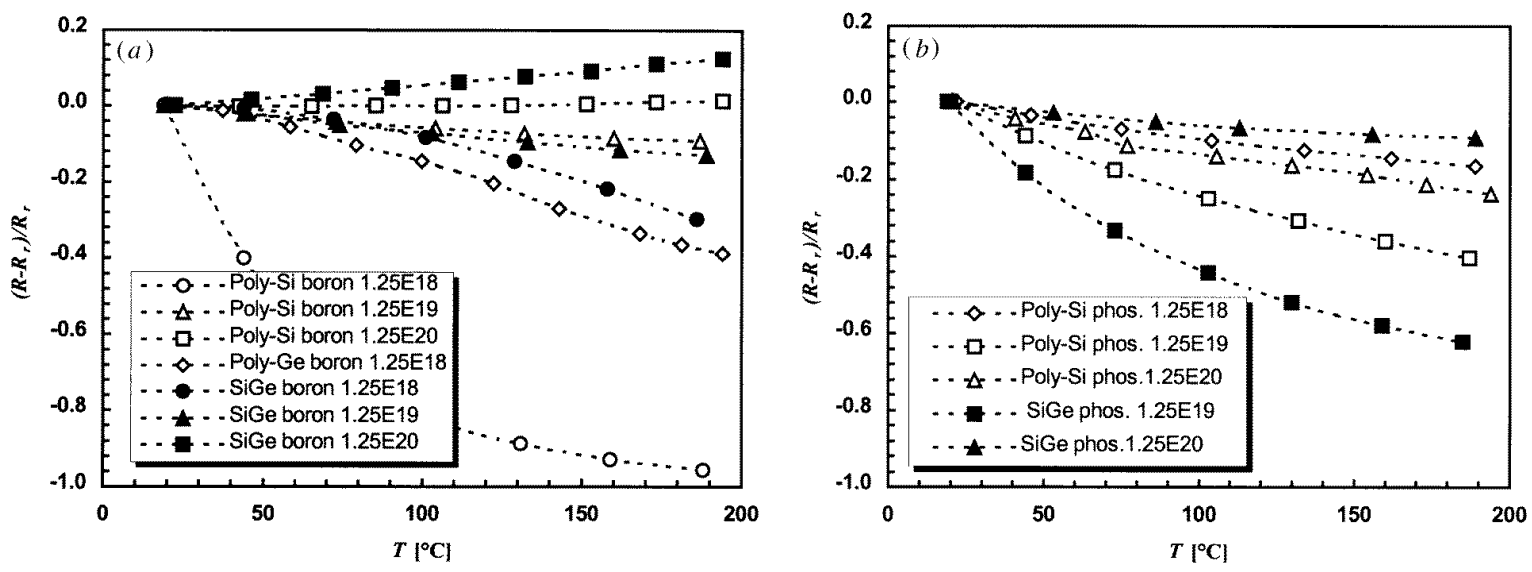

Figure 2. Dependence of resistivity on temperature for: (a) boron-doped, and $(b)$ phosphorus-doped sensors.

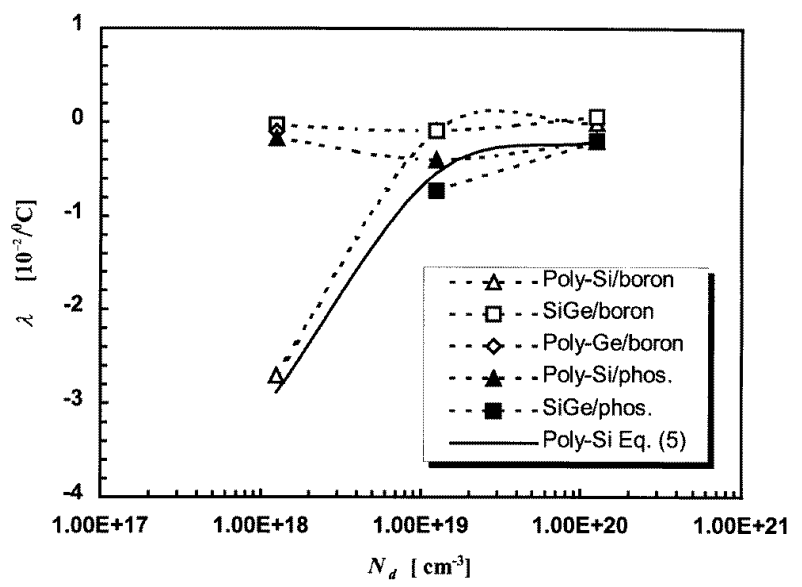

Figure 3. Dependence of TCR on doping concentration.

requirements, bridge-like thermistors are constructed on moderately doped films. Figure 4 shows the two ends of a bridge, serving as signal leads, are heavily doped to give low-temperature sensitivity and low electrical resistivity. The 'sensing' region at the centre is moderately doped for optimal parameter control, temperature sensitivity and operational stability. It is also made small for high spatial resolution.

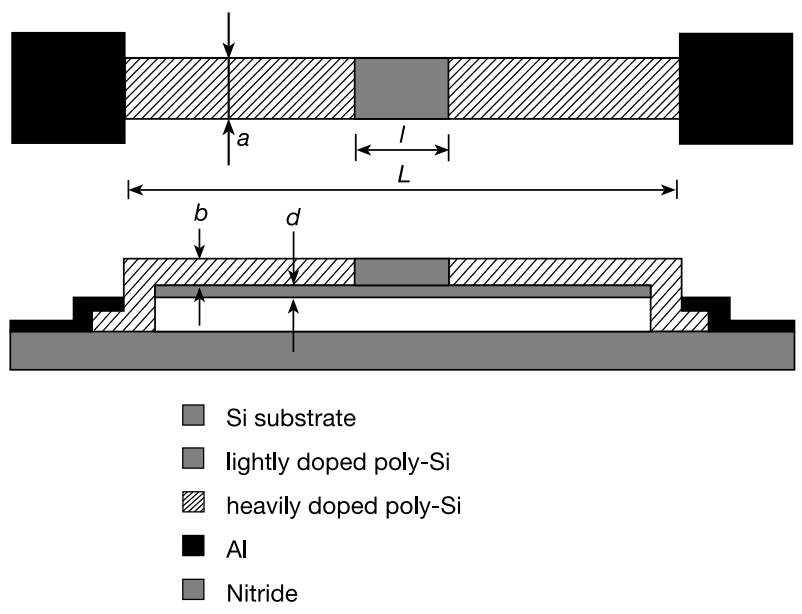

Figure 4. Schematic diagram of the sensor design.

A variety of thermistors with different sizes and cavity structures were fabricated. The starting substrates were $100 \mathrm{~mm}\langle 100\rangle$ oriented $n$-type silicon wafers with thickness of $550 \mu \mathrm{m}$ and resistivity of about $4-7 \Omega \mathrm{cm}^{-1}$. The fabrication began with the formation of an electrically and thermally insulating stack of $0.2 \mu \mathrm{m}$ thermal oxide at $950{ }^{\circ} \mathrm{C}$, 
underneath a $0.1 \mu \mathrm{m}$ low-stress silicon nitride film deposited at $840^{\circ} \mathrm{C}$ in a low-pressure chemical vapour deposition (LPCVD) reactor. A $1 \mu \mathrm{m}$ low-temperature oxide (LTO) layer was deposited at $425^{\circ} \mathrm{C}$ at low pressure over the nitride layer and was patterned to be the sacrificial layer for the air cavities that would be formed. After the deposition of $0.7 \mu \mathrm{m}$ low-stress silicon nitride, etching holes were opened. This nitride layer would form the structural frames over the cavities. A $0.4 \mu \mathrm{m}$ layer of undoped polycrystalline semiconducting material: $\mathrm{Si}, \mathrm{Ge}$ or $\mathrm{Si}_{0.8} \mathrm{Ge}_{0.2}$, was deposited by LPCVD under 300 mtorr pressure at temperatures of 620 , 350 or $550^{\circ} \mathrm{C}$, respectively. The films were then patterned into 'dumbbell'-shaped thermistor bridges. The entire film first had been moderately doped by boron with $60 \mathrm{KeV}$ or phosphorus with $160 \mathrm{KeV}$ implantation before the 'lead' portions of the bridges were selectively heavily implanted. Two dosage levels were selected for the moderate doping of both species, either $5 \times 10^{13}$ or $5 \times 10^{14} \mathrm{~cm}^{2}$, while the dosage level for the heavy doping was in all cases $5 \times 10^{15} \mathrm{~cm}^{2}$. The doped films: $\mathrm{Si} \mathrm{Ge}$ or $\mathrm{Si}_{0.8} \mathrm{Ge}_{0.2}$ were then annealed at $900^{\circ} \mathrm{C}$ for $30 \mathrm{~min}, 600^{\circ} \mathrm{C}$ for $1 \mathrm{~h}$ or $850^{\circ} \mathrm{C}$ for $1 \mathrm{~h}$, respectively. After the sacrificial LTO layer was etched away in $30 \mathrm{wt} \%$ buffered oxide etch solution, thermally insulating air cavities resulted from the remaining nitride frames. A layer of LTO with a thickness of $0.8 \mu \mathrm{m}$ was deposited at $425^{\circ} \mathrm{C}$ and patterned to protect the sensing part of each thermistor, as well as to define the metal contact areas. Finally, Al-Si alloy was sputter deposited, patterned, and sintered at $450{ }^{\circ} \mathrm{C}$ for $30 \mathrm{~min}$ in forming gas to provide lowresistance electrical contacts. Photographs of three typical temperature microsensors with different cavity designs are shown in figure 5.

For accurate extraction of device parameters, the process-induced (lithography, dopant diffusion, etching etc) dimensional changes, $l_{i}$, in the sensors must be determined. For example, the diffusion of dopants from the heavily doped leads to the moderately doped sensing region effectively decreases the actual length of the sensing region, and with it the overall resistance of the bridge. The extracted dimensional reduction is summarized in figure 6 . As expected, the overall bridge length and width has a small effect on the dimensional change of the lightly doped region which is found to be about $1 \mu \mathrm{m}$. This means that the diffusion length of dopants on each side of the sensor is about $0.5 \mu \mathrm{m}$.

\section{Bridge analysis}

When current passes through a sensor, electrical power is dissipated and converted into heat by Joule's heating within the sensor. The differential thermal power, $\mathrm{d} P$, generated in a different element, $\mathrm{d} x$, is given by:

$$
\begin{aligned}
& \frac{\mathrm{d} P}{\mathrm{~d} x}=J^{2} \chi a b \\
& \quad=J^{2} \chi_{0} a b\left[1+\lambda_{1}\left(T-T_{0}\right)+\lambda_{2}\left(T-T_{0}\right)^{2}+\cdots\right] \\
& \quad \approx J^{2} \chi_{0} a b\left[1+\lambda_{1}\left(T-T_{0}\right)\right]
\end{aligned}
$$

where $a$ and $b$ are the width and height of the bridge, $J$ is the current density, $\chi$ is the resistivity, and $\lambda_{1}, \lambda_{2}$, etc, are the

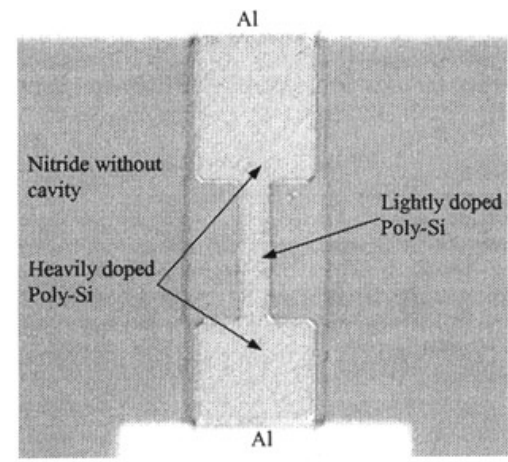

(a)

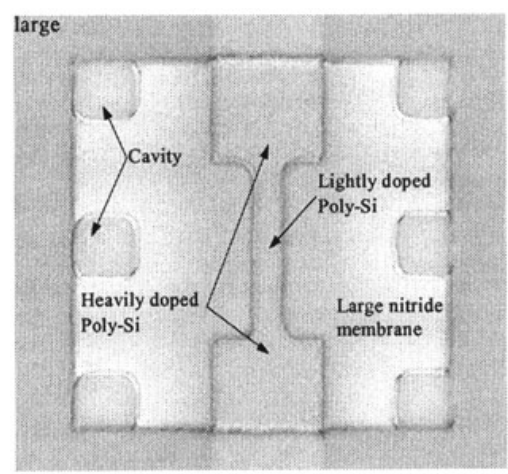

(b)

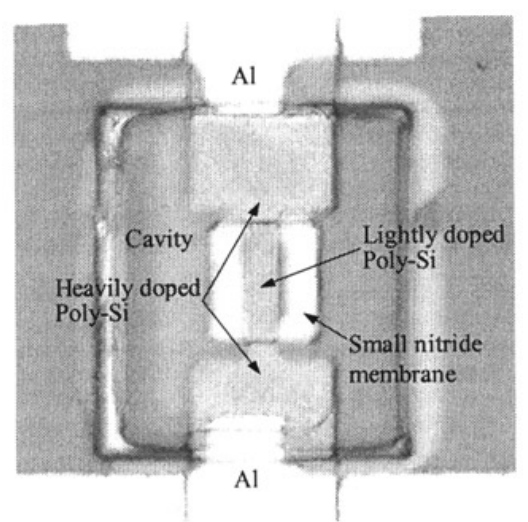

(c)

Figure 5. Illustrations of three different configurations. (a) Sensor on substate, (b) sensor on a large membrane, $(c)$ sensor on a small membrane.

temperature coefficients of resistance; $\chi_{0}$ is the resistivity at a chosen reference temperature, $T_{0}$. A fraction of this power is stored as internal energy, thus increasing the sensor temperature, and the rest is transferred to the surrounding. The time rate of change of the differential internal energy, $\mathrm{d} \dot{Q}$, stored in the differential element is:

$$
\frac{\mathrm{d} \dot{Q}}{\mathrm{~d} x}=\rho_{b} c_{b} a b \frac{\partial T}{\partial t}
$$

where $\rho_{b}$ and $c_{b}$ are the mass density and the specific heat capacity of the bridge material, respectively. If the sensor temperature is different from that of the surrounding, heat will be transferred from the sensor to the surrounding. Three heat transfer mechanisms can be identified: conduction, 

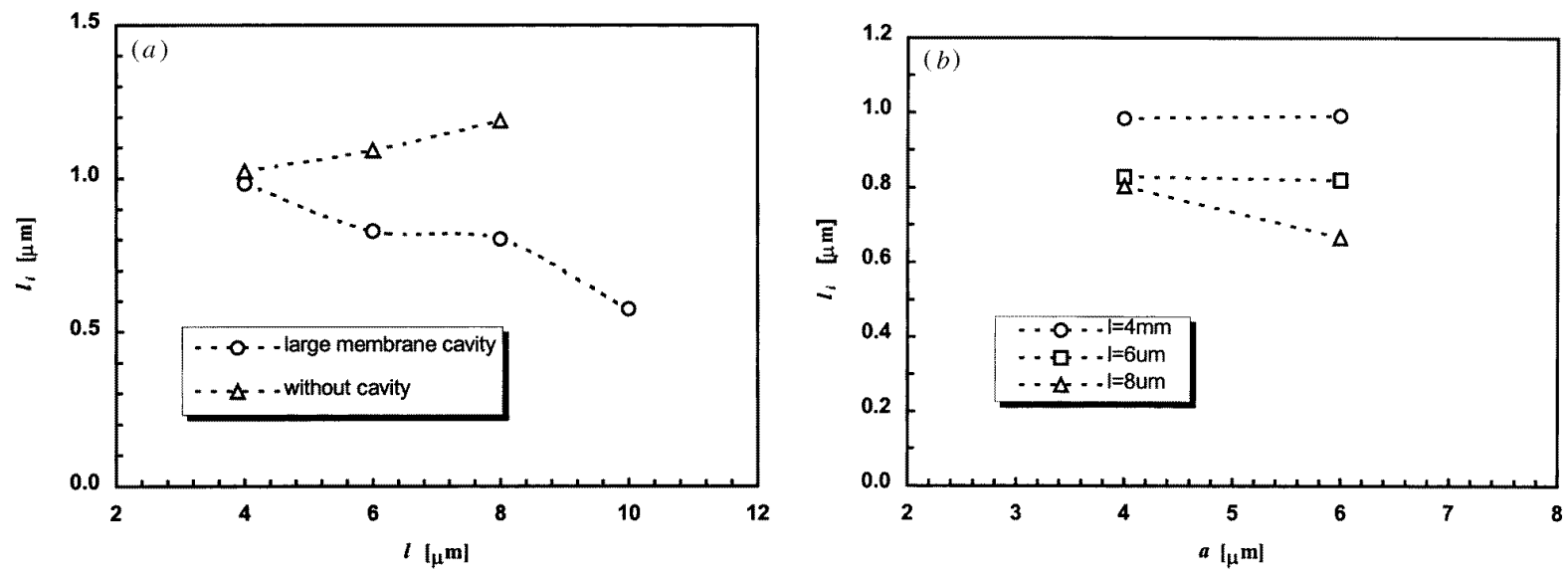

Figure 6. Dependence of the process-induced dimensional change on: $(a)$ length, and $(b)$ width of moderately doped region.

convection and radiation. The heat conduction in a differential elements, $\mathrm{d} \dot{q}_{d}$, takes place either through the sensor body and the electrical leads to the substrate, or through the bottom nitride layer on which the sensor is resting to the substrate, thus:

$$
\frac{\mathrm{d} \dot{q}_{d}}{\mathrm{~d} x}=-k_{b} a b \frac{\partial T^{2}}{\partial x^{2}}+k_{N} a \frac{T-T_{w}}{d}
$$

where $k_{b}$ is the thermal conductivity of the bridge, $k_{N}$ is the thermal conductivity of the insulating nitride layer and $d$ is its thickness; $T_{w}$ is the substrate temperature. However, in the presence of a gap between the nitride layer and the substrate, the equation needs to be suitably modified:

$$
\frac{\mathrm{d} \dot{q}_{d}}{\mathrm{~d} x}=-k_{b} a b \frac{\partial^{2} T}{\partial x^{2}}+k_{N} a \frac{T-T_{g}}{d}
$$

where $T_{g}$ is the ambient gas temperature. Natural or forced heat convection, $\mathrm{d} \dot{q}_{h}$, takes place through the upper and the two side surfaces of the differential element which are in contact with the ambient gas:

$$
\frac{\mathrm{d} \dot{q}_{h}}{\mathrm{~d} x}=h(a+2 b)\left(T-T_{g}\right)
$$

where $h$ is the heat-transfer coefficient is related to the Nusselt number, $N u$, as follows

$$
N u=\frac{h D_{h}}{k_{g}}
$$

$D_{h}$ is the bridge hydrodynamic diameter and $k_{g}$ is the thermal conductivity of the ambient gas. The Nusselt number for the bridge is given by (Churchill and Bernstein 1977):

$$
N u=0.3+\frac{0.62 \sqrt{R e} \cdot \operatorname{Pr}^{1 / 3}}{\left[1+(0.4 / P r)^{2 / 3}\right]^{1 / 4}}
$$

where $\mathrm{Pr}$ is the Prandtl number of the ambient gas.

Heat is also dissipated to the environment by radiation, $\mathrm{d} \dot{q}_{r}$, through the same upper and side surfaces of the element:

$\frac{\mathrm{d} \dot{q}_{r}}{\mathrm{~d} x}=\sigma_{b}(a+2 b)\left(T^{4}-T_{g}^{4}\right) \approx 4 \sigma_{b}(a+2 b) T_{g}^{3}\left(T-T_{g}\right)$ where $\sigma_{b}$ is the Stefan-Boltzmann coefficient of the bridge. Therefore, the heat balance at any point along the bridge is given by:

$$
\begin{aligned}
& \frac{1}{\alpha_{b}} \frac{\partial T}{\partial t}=\frac{\partial^{2} T}{\partial x^{2}}-\beta\left(T-T_{g}\right)-\gamma\left(T-T_{w}\right) \\
& \quad+\delta\left[1+\lambda_{1}\left(T-T_{0}\right)\right]
\end{aligned}
$$

where

$$
\begin{gathered}
\alpha_{b}=\frac{k_{b}}{c_{b} \rho_{b}} \\
\beta=\frac{h}{k_{b}}\left(\frac{2}{a}+\frac{1}{b}\right)+\frac{4 \sigma_{b} T_{g}^{3}}{k_{b}}\left(\frac{2}{a}+\frac{1}{b}\right) \\
\gamma=\frac{k_{N}}{k_{b}} \frac{1}{b d} \quad \delta=\frac{J^{2} \chi_{0}}{k_{b}} .
\end{gathered}
$$

Equation (14) is rewritten as follows

$$
\frac{1}{\alpha_{b}} \frac{\partial T}{\partial t}=\frac{\partial^{2} T}{\partial x^{2}}-\varepsilon\left(T-\frac{\psi}{\varepsilon}\right)
$$

where

$\varepsilon=\beta+\gamma-\delta \lambda_{1} \quad \psi=\beta T_{g}+\gamma T_{w}+\delta\left(1-\lambda_{1} T_{0}\right)$.

This governing equation can be solved by separation of variables with the proper boundary and initial conditions.

\subsection{Steady-state response}

The steady-state temperature distribution along the bridge can be calculated by dropping the time-dependent term in equation (7). However, the equation has to be solved twice for regions I (moderately doped) and II (heavily doped), since the resistivity in the two regions is about an order of magnitude apart. In order to simplify the solution, we can select: $T_{w}=T_{g}=T_{0}$. This case corresponds to a situation where the wall and the bridge reference temperature is equal to the ambient gas temperature. The equation now reads:

$$
\frac{\partial^{2} T}{\partial x^{2}}-\varepsilon\left(T-\frac{\phi}{\varepsilon}\right)=0
$$


L Jiang et al

where $\phi=\varepsilon T_{0}+\delta$. The proper boundary conditions are:

$$
\begin{gathered}
\frac{\partial T_{I}}{\partial x}(x=0)=0 \\
T_{I}\left(x=\frac{l}{2}\right)=T_{I I}\left(x=\frac{l}{2}\right) \\
\frac{\partial T_{I}}{\partial x}\left(x=\frac{l}{2}\right)=\frac{\partial T_{I I}}{\partial x}\left(x=\frac{l}{2}\right) \\
T_{I I}\left(x=\frac{L}{2}\right)=T_{0}
\end{gathered}
$$

where $l$ is the length of the centre, moderately doped region, and $L$ is the length of the entire bridge including both the moderately doped sensing region and the heavily doped leads regions. The solution for the two regions is:

$$
T_{I}(x)=-A l_{c l}^{2}+C_{1}\left(\mathrm{e}^{x_{I} / l_{c I}}+\mathrm{e}^{-x_{I} / l_{c I}}\right)
$$

and

$$
T_{I I}(x)=-A_{I I} l_{c I I}^{2}+C_{2} \mathrm{e}^{x_{I I} / l_{c I I}}+C_{3} \mathrm{e}^{-X_{I I} / L_{C I I}}
$$

where

$$
A_{i}=\frac{J^{2} \chi_{i}}{k_{b}} \quad l_{c i}=\left(\frac{2(a+b) h}{a b k_{b}}-J^{2} \chi_{i} \frac{\lambda_{i}}{k_{b}}\right)^{-\frac{1}{2}} .
$$

Here, $i=I$ for the lightly doped and $i=I I$ for the heavily doped region. The coefficients $C_{1}, C_{2}$ and $C_{3}$ can be obtained by imposing the boundary conditions. The material properties such as the specific heat capacity, thermal conductivity and mass density are assumed to be the same for the moderately and heavily doped regions. The resulting temperature distributions due to different current levels for a poly-Si bridge are plotted in figure 7. As expected, the temperature increases everywhere along the bridge as the current increases. Also, the temperature at the moderately doped centre region of the bridge is the highest since most of the heat is generated there. It is difficult to directly measure the temperature distribution along the bridge. However, utilizing the resistivity-temperature data, the bridge resistivity distribution due to the temperature distribution can be calculated. The results are shown in figure 8 . The resistivity at the centre is about an order of magnitude higher than the two heavily doped leads of the bridge due to the different doping levels. The resistance of the entire bridge can be calculated for any given current by integrating the resistivity as follows

$$
R=\int_{-L / 2}^{+L / 2} \frac{\chi(x)}{a b} \mathrm{~d} x .
$$

The voltage drop across the bridge for any given current, $I$, is simply given by:

$$
V=I R \text {. }
$$

These calculated values are compared in figure 9 with direct $I-V$ curve measurements obtained by Tektronic 370A programmable curve tracer. The agreement between the calculations and the measurements is satisfactory. The deviation at high current is probably due to the exclusion of

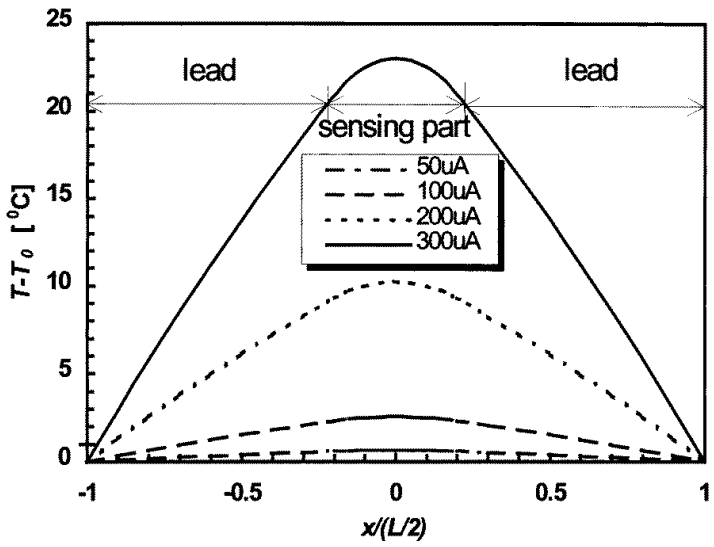

Figure 7. Temperature distribution due to current heating.

the higher-order TCRs when formulating the Joule's heating term in equation (18). Nevertheless, the first-order TCR model yields reliable results for current levels below $1 \mathrm{~mA}$. Hence, it will be used to study the bridge transient response.

It is interesting to compare the contribution of each heat transfer mechanism with the steady-state temperature distribution. Figure 10 compares the heat transfer rates from the bridge with the surrounding by conduction, convection and radiation for a reasonable current range. The heat loss due to convection is less than $0.3 \%$ of the heat loss due to radiation does not exceed $0.03 \%$ of the heat loss due to convection. Consequently, the radiation term will be neglected in the subsequent analysis, as was done by others.

\subsection{Transient response}

In order to study the unsteady behaviour of the bridge, equation (7) has to be solved with the proper boundary and initial conditions. Again, for simplicity we shall assume $T_{w}=T_{g}=T_{0}$, neglect radiation and utilize the same boundary conditions (equation (9)) for all times. The initial condition is uniform temperature everywhere before a step current is applied to the bridge:

$$
T_{I}(x, t=0)-T_{I I}(x, t=0)=T_{0} .
$$

Mastrangello (1990) has shown that for a uniformly doped bridge with a fixed temperature at both ends, the solution is a multimode response, and the eigenvalue of the first mode determines the time constant:

$$
\tau_{1}=\tau_{1 d} \tau_{h} /\left(\tau_{1 d}+\tau_{h}\right)
$$

where

$$
\tau_{1 d}=\frac{\rho_{b} c_{b}}{k_{b}}\left(\frac{l^{2}}{\pi^{2}}\right) \quad \tau_{h}=\frac{\rho_{b} c_{b}}{\lambda_{1} J^{2} \chi_{0}-2 h \frac{(a+b)}{a b}} .
$$

It can be seen from the above expressions that the time constant is mainly due to two components: a conduction time constant, $\tau_{1 d}$ which has an $l^{2}$ dependence and therefore is small and dominates for short bridges, and a convection time constant, $\tau_{h}$, which dominates for long bridges. In our 


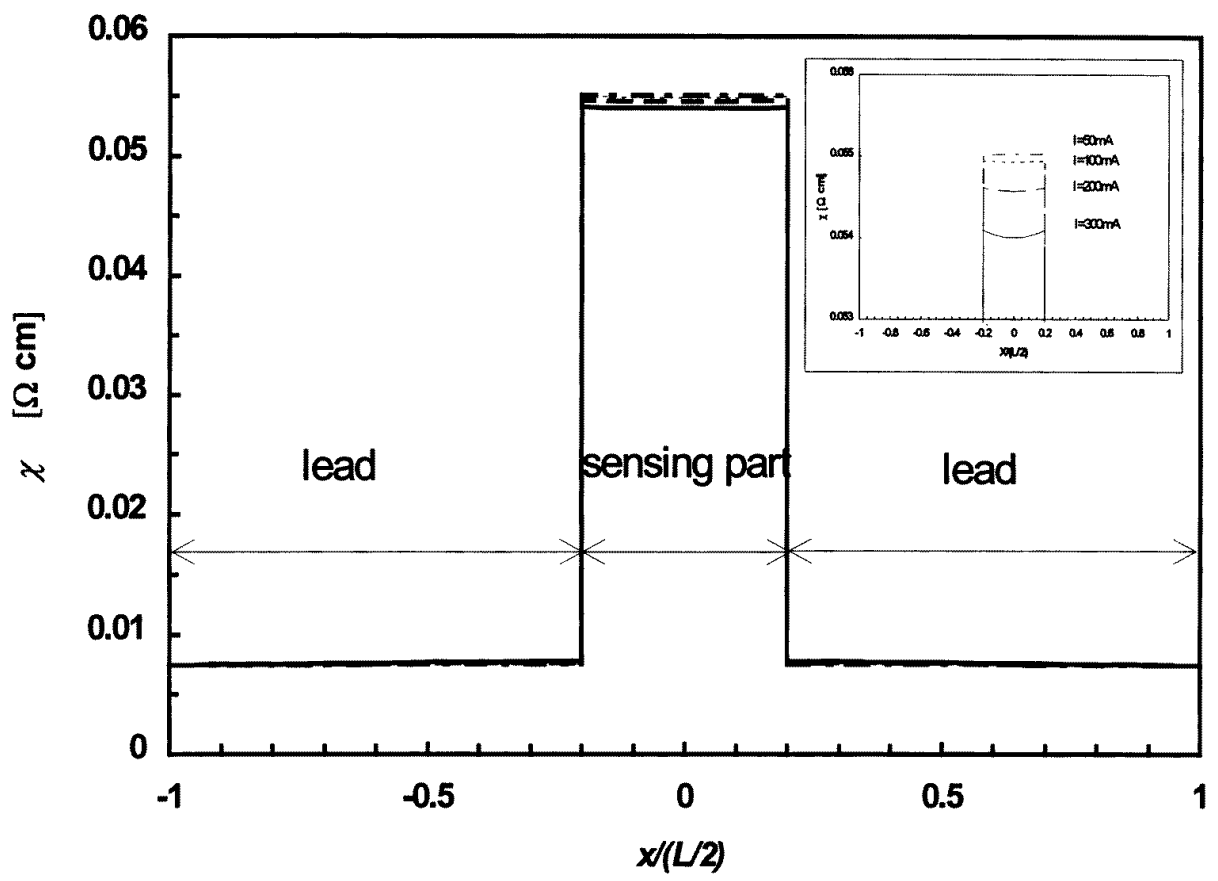

Figure 8. Resistivity distribution due to current heating.

case, we expect the conduction time constant to dominate, namely $\tau_{1 d}<\tau_{h}$, and thus

$$
\tau_{1}=\frac{\tau_{1 d} \tau_{h}}{\tau_{1 d}+\tau_{h}} \sim \frac{\tau_{1 d} \tau_{h}}{\tau_{h}}=\tau_{1 d}
$$

It should be noted that this is not a first-order system due to the conduction mechanism which introduces a higher-order term. Therefore, more than one time constant may exist. For example, the second time constant associated with the second mode is:

$$
\tau_{2}=\tau_{2 d} \tau_{h} /\left(\tau_{2 d}+\tau_{h}\right)
$$

where

$$
\tau_{2 d}=\frac{\rho_{b} c_{b}}{k_{b}}\left(\frac{l^{2}}{4 \pi^{2}}\right) \quad \tau_{h}=\frac{\rho_{b} c_{b}}{\lambda_{1} J^{2} \chi_{0}-2 h \frac{(a+b)}{a b}} .
$$

Notice that the convection time is unchanged, since the terms involved enter the governing equation as first-order system. However, the conduction time constant has changed as this term is of higher order. In the present bridge design, the combined contribution of the moderately and heavily doped region to the time constant is not trivial. Jiang et al (1994) suggested using the same expressions with an effective length of the bridge, $l_{e}$, which should be longer than $l$ and smaller than $L$.

The time constant is experimentally determined by passing a step current through the bridge in still air and observing the voltage rise of fall in an oscilloscope. Figure 11 shows typical voltage waveform responses of a bridge with negative TCR to a step current. The initial rise time in the heating process, figure 11(a), is due to the electrical time constant as it takes a finite time for the current to increase from zero to the desired value. Initially, the bridge is still cold and its resistance is high, therefore, the voltage output

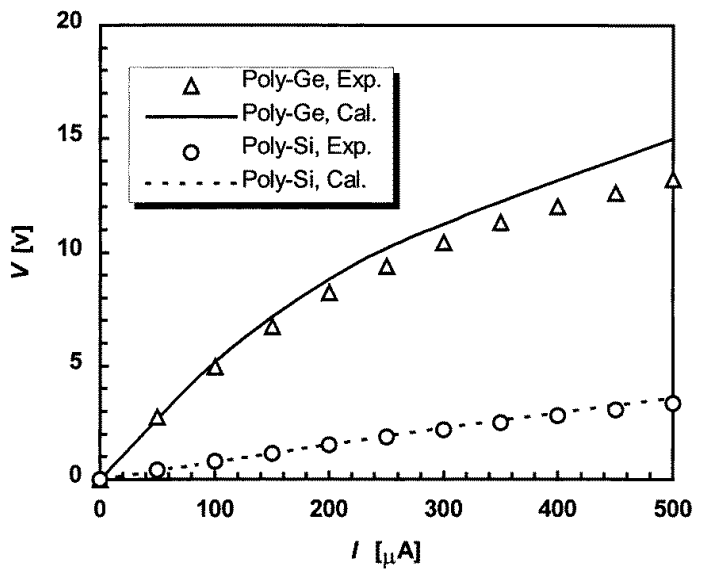

Figure 9. $I-V$ characteristics.

attains its maximum value. However, as the electrical power is dissipated into heat, the thermal time constant comes into play since the bridge temperature increases and its resistivity decreases. The current is kept constant, thus the voltage output drops from its peak to a steady-state value. A similar behaviour is observed in the cooling process when the current is turned off, as shown in figure 11(b). However, if the voltage output drops to zero, i.e. no bias voltage, the voltage overshoot is not as prominent as in the heating process.

Although the time constant and the frequency response are closely related, the frequency response in many instances is of more interest. Both depend on material properties: $\rho_{b}, C_{b}, k_{b}, \lambda, \chi_{0}$, geometry: $a, b, l$ and operating conditions: $J, h(R e)$. Figure 12 depicts the frequency response of various bridge structures. It is expected that the effective length of the bridge will increase as the length of the centre moderately doped region increases. The dominant 


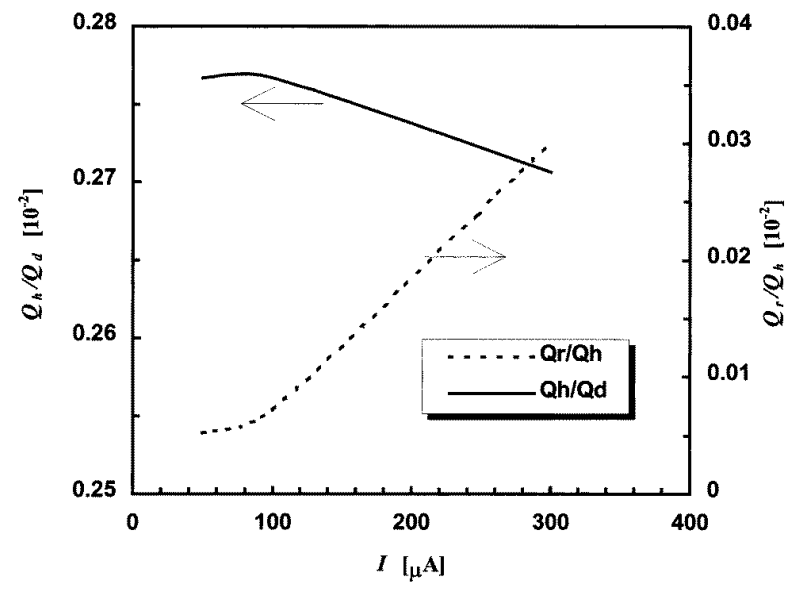

Figure 10. The relative contribution of radiation, convection and conduction heat transfer from the sensor to the ambient (poly-Si, $20 \times 0.4 \mu \mathrm{m}^{3}$, boron doped, $1.25 \times 10^{19} \mathrm{~cm}^{-3}$ ).
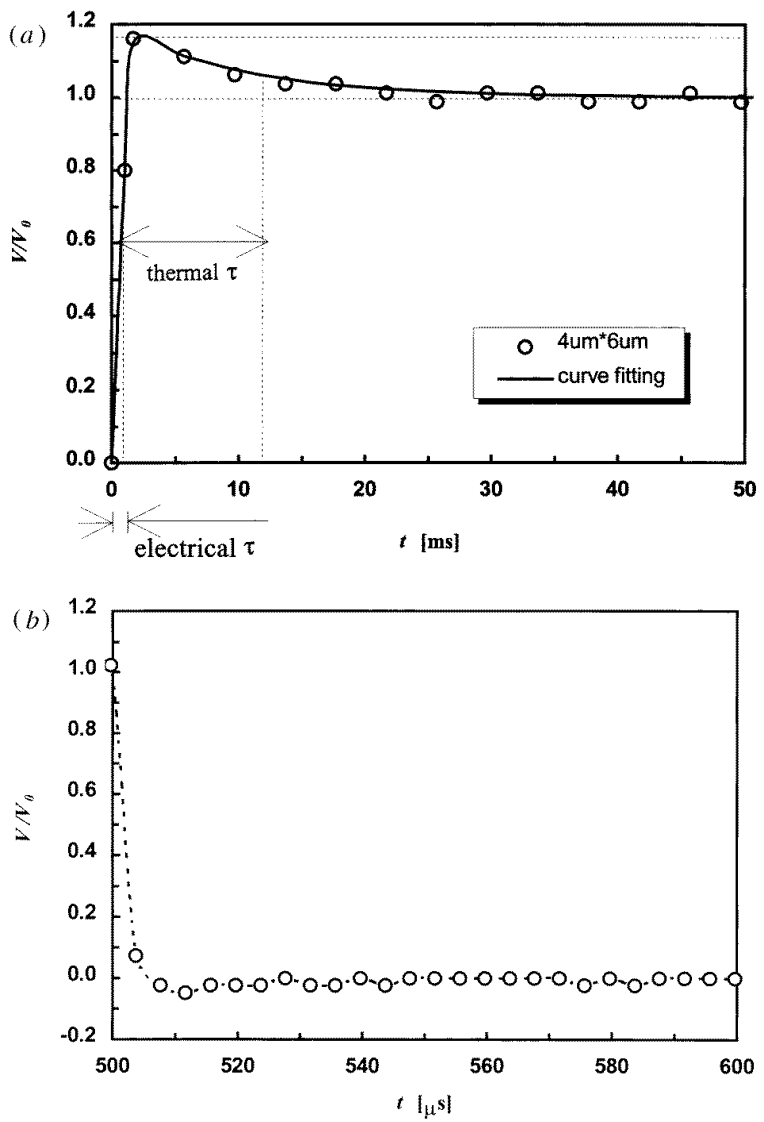

Figure 11. Bridge voltage response to a step current input: (a) heating, and $(b)$ cooling response (SiGe sensor, phosphorus doped, $\left.1.25 \times 10^{19} \mathrm{~cm}^{-3}\right)$.

conduction time constant will then increase as well as leading to a slower response. Indeed, figure 12(a) shows that the corner frequency for poly-Si bridges increases, indicating a smaller time constant, as the length of the moderately doped region decreases. Figure 12(b) compares the frequency response of three poly-Si bridges with different configurations. The bridge constructed directly on the silicon substrate, without an air gap, has the slowest frequency
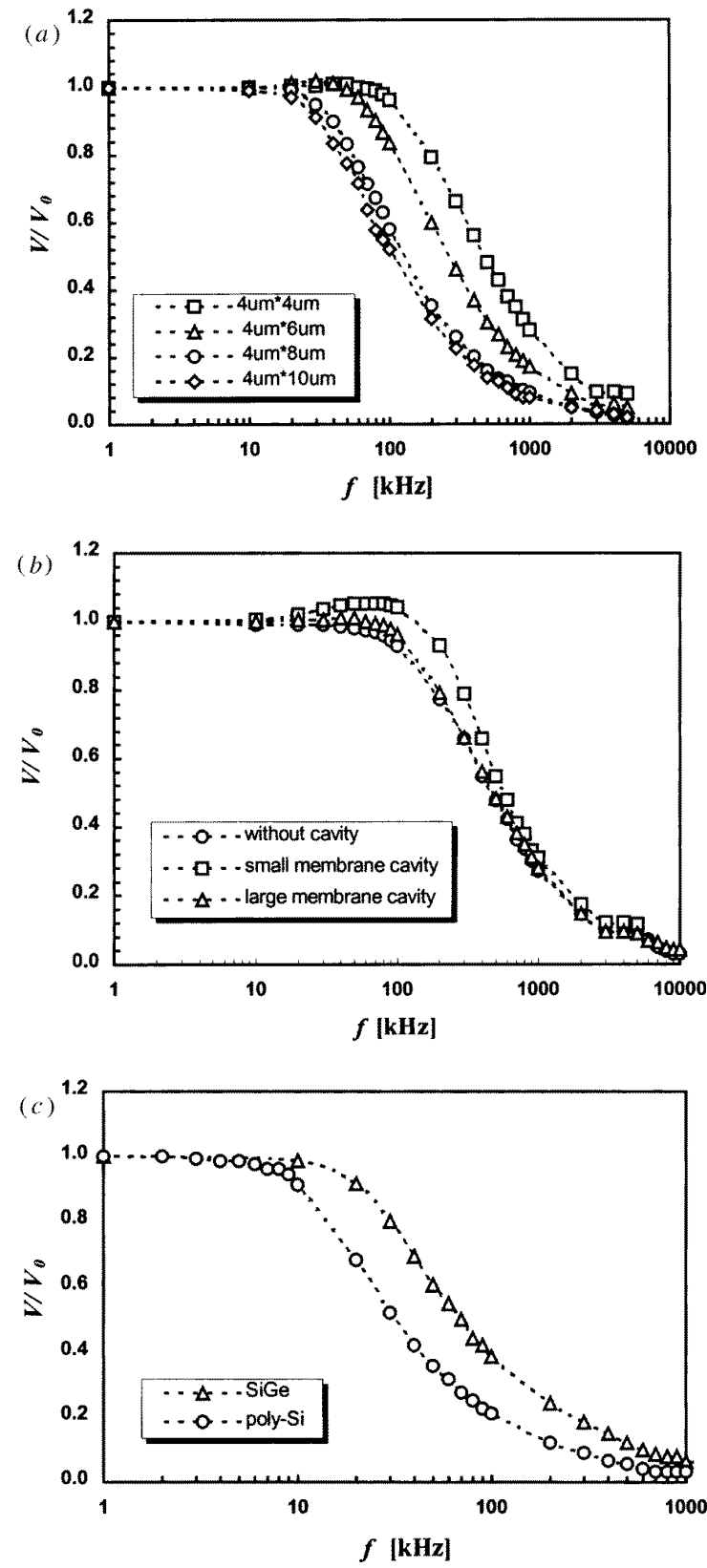

Figure 12. Frequency response for bridge with different: $(a)$ moderately doped length, $(b)$ cavity structure, and (c) materials.

response since its thermal mass is the highest. The air-gap bridge with the smaller nitride membrane has the smallest thermal mass. Hence, its corner frequency is the highest. It is true that the differences between the three configurations are not significant. Figure 12(c) compares poly-Si and poly-SiGe bridges having identical geometry, doping and operating conditions. It is clear that poly-SiGe has the faster time response, and it is mainly due to a larger thermal diffusivity, $\alpha_{b}$.

\section{Sensor applications}

So far, for simplicity, we have analysed the bridge performance in still air with the assumption that the substrate 


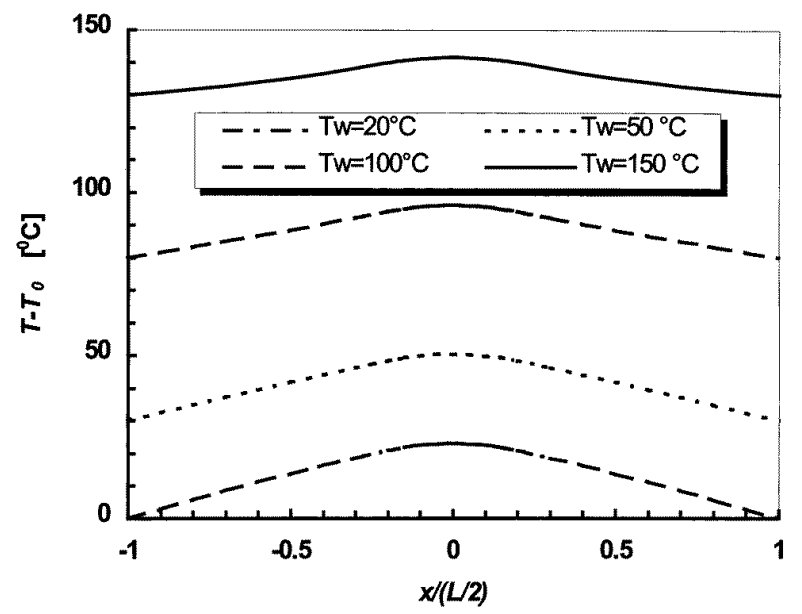

Figure 13. Temperature distribution for different wall temperature (poly-Si, boron doped, $1.25 \times 10^{19} \mathrm{~cm}^{-3}, 4 \times 4 \mu \mathrm{m}^{2}$, $I=300 \mu \mathrm{A}, R e=0)$

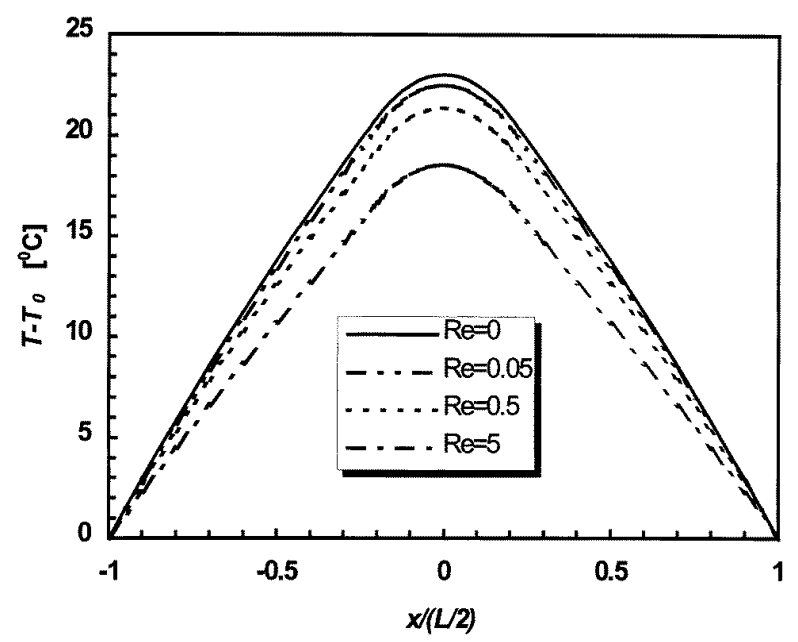

Figure 14. Temperature distribution for different $R e$ (poly-Si, boron doped, $1.25 \times 10^{19} \mathrm{~cm}^{-3}, 4 \times 4 \mu \mathrm{m}^{2}, I=300 \mu \mathrm{A}$, $\left.T_{w}=20^{\circ} \mathrm{C}\right)$.

temperature is equal to the ambient gas temperature. Of course, in a real application that is not the case; the gas temperature may be different from the substrate temperature and the gas velocity need not be zero. Figure 13 depicts the temperature distribution over the bridge in still air and driven by a given current, $300 \mu \mathrm{A}$, for different substrate temperatures. Since the conduction mechanism is so dominant, the bridge temperature everywhere increases if the substrate temperature increases. The relative contribution of the Joule's heating decreases and, thus, the temperature gradient along the bridge also decreases as the substrate temperature increases. Figure 14 shows the effect of the gas velocity, $U$, or the Reynolds number, $R e=U D_{h} / v$, where $v$ is the gas kinematic viscosity. The current level is constant, $300 \mu \mathrm{A}$, and the substrate temperature equals the gas temperature of $20^{\circ} \mathrm{C}$. The convection mechanism is very small compared with conduction and thus a large increase in the $R e$ is required, from 0.005 to 5 , in order to lower the maximum bridge temperature by $8^{\circ} \mathrm{C}$. Figure 15 shows that for the same material, poly-Si, and under the same

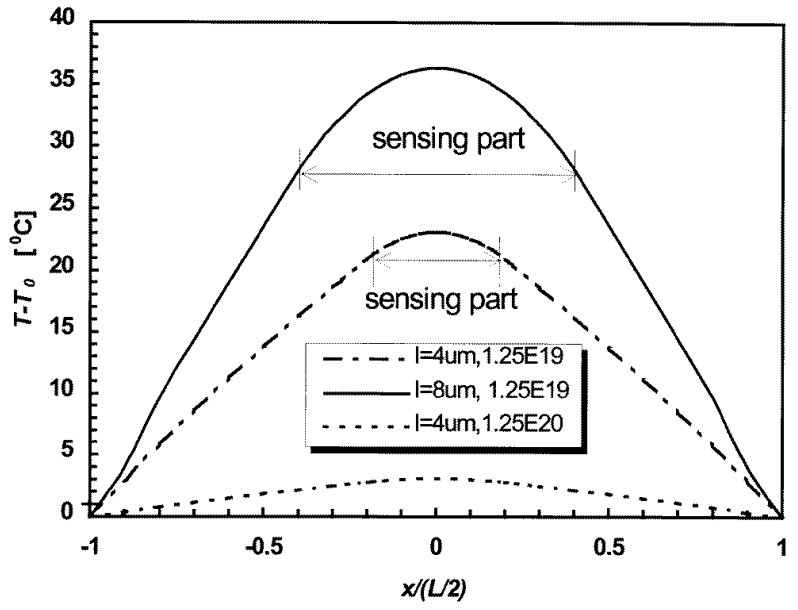

Figure 15. Temperature distribution for different sensing part length and doping concentration (poly-Si, $I=300 \mu \mathrm{A}$, $T_{w}=20^{\circ} \mathrm{C}$ ).

operating conditions, the longer is the moderately doped region the higher is the temperature everywhere along the sensor. This is expected since the total power dissipation increases due to a longer high-resistivity region. Similarly, when the doping concentration increases and the resistivity decreases, the resulting temperature distribution decreases if all other parameters are the same.

The temperature of the centre, moderately doped region is the parameter which dominates the overall bridge resistance and subsequently the bridge voltage output. Figure 16 summarizes the change of the maximum bridge temperature, $T(x=0)=T_{\max }$, when either the gas velocity or the substrate temperature changes. It is interesting to note that the maximum bridge temperature, for $R e<0.1$, does not drop more than $10 \%$ of the maximum temperature in still air, $R e=0$, regardless of the substrate temperature. This means that if a substantial temperature change is required, namely more than $10 \%$, a much higher $R e$ is required as shown in figure 16(a). Indeed, figure $16(b)$ shows that the relative change of the maximum bridge temperature is not sensitive to the substrate temperature. This is again the result of the strong dominance of the conduction mechanism in transferring heat from the bridge to the substrate as compared with the heat convection or radiation from the bridge to the ambient gas.

The steady-state temperature distribution along the bridge (equation (2)), gives rise to a characteristic length scale, $l_{c}$. This length scale depends not only on the bridge dimensions, but also on the material properties and on the operating conditions. Thus, the bridge temperature strongly depends on $l_{c}$. Figure 17 shows the effect of the characteristic length scale on the normalized maximum temperature of the bridge. The bridge maximum temperature approaches the wall temperature when either the bridge length, $L$, approaches zero or the characteristic length scale, $l_{c}$, approaches infinity. In order to obtain higher sensitivity from the bridge, namely larger separation between the bridge and the wall temperature, either $L$ should be very large or $l_{c}$ should be very small. 

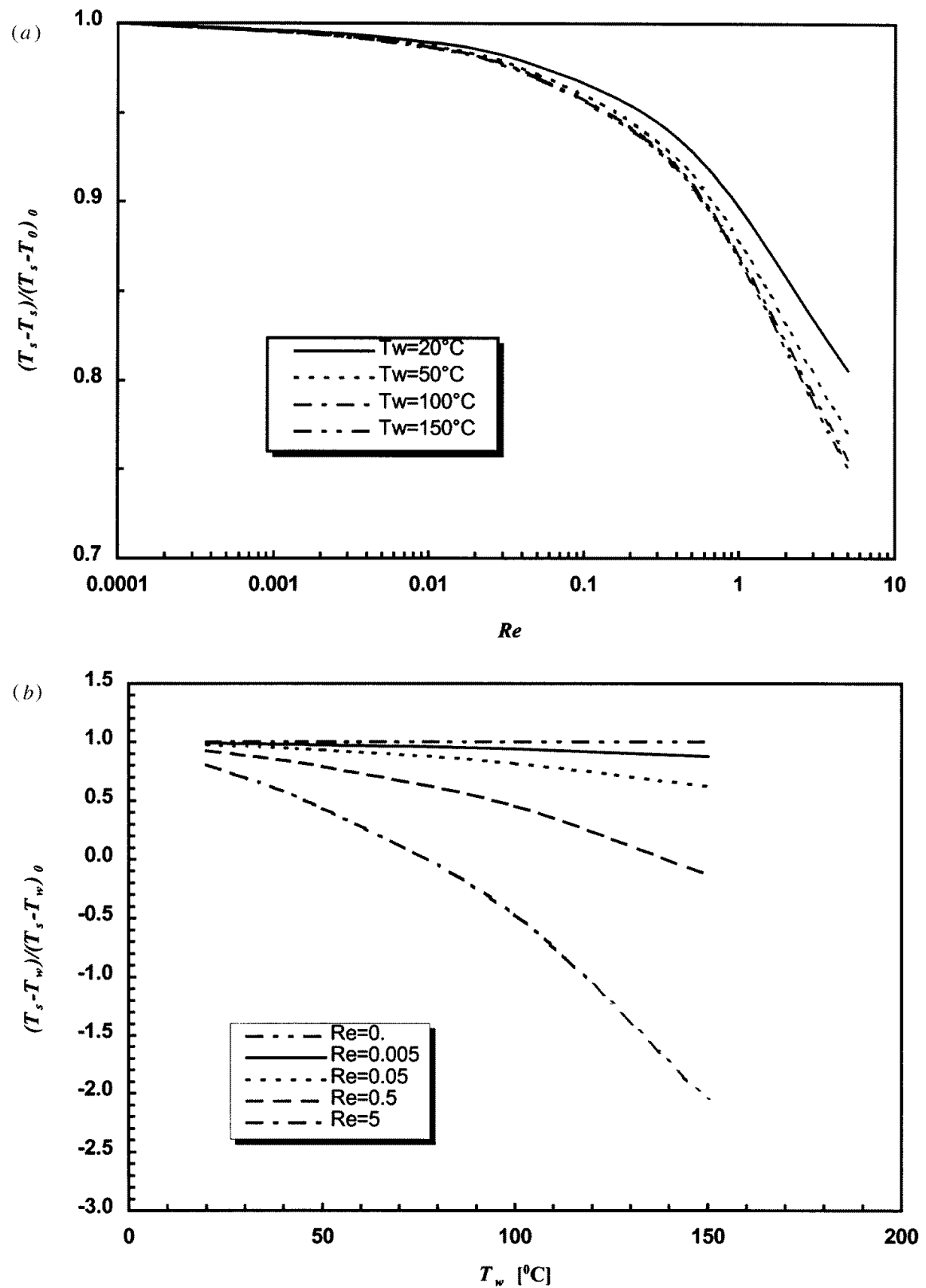

Figure 16. Dependence of sensor temperature on (a) Reynolds number, and (b) wall temperature (poly-Si, $4 \times 4 \mu \mathrm{m}^{2}$, boron doped, $\left.1.25 \times 10^{19} \mathrm{~cm}^{-3}, I=300 \mu \mathrm{A}\right)$.

An important aspect of a sensor operation is its stability (drift) during operation and its repeatability over an extended period of time. Resistors based on heavily doped polycrystalline materials should demonstrate a high level of long-term stability since surface effect plays only a minor role in device characteristics, especially with passivation. Obermeier (1986) reported that at a temperature of $125^{\circ} \mathrm{C}$ over a time period of $1000 \mathrm{~h}$ a drift of less than 0.005 was obtained. However, this was for uniformly doped poly-Si resistors. In the present bridge design, the stability may not exhibit similar performance as sharp boundaries between the moderately and highly doped regions exist, and as a result, the sensor performance may change over time. Indeed, burnin has become an important tool in improving the quality of semiconductor devices (Wood et al 1993). Two approaches in the burn-in procedure can be effective for the present sensor design: temperature cycling (TC) and duty cycling (DC). In the TC approach, the sensor ambient temperature varies in an appropriate range, and a time period of about $30 \mathrm{~min}$ is required for each temperature. This process should be repeated 5-10 times. In the DC approach, an appropriate current is applied to the sensor for a long time. A time period of more than $100 \mathrm{~h}$ is typically required. Of course, if both approaches are combined simultaneously, the equivalent hours can be compressed into a shorter period of time. Figure 18 shows calibration curves of poly-SiGe bridges after a burn-in procedure of five cycles $\mathrm{TC}$ at a temperature range of $20-200^{\circ} \mathrm{C}$ and $50 \mathrm{~h} \mathrm{DC}$ with $50 \mu \mathrm{A}$. The difference between the calibration curves of three different sensors before and after $24 \mathrm{~h}$ delay without operation (figure $18(a)$ ), is less than 


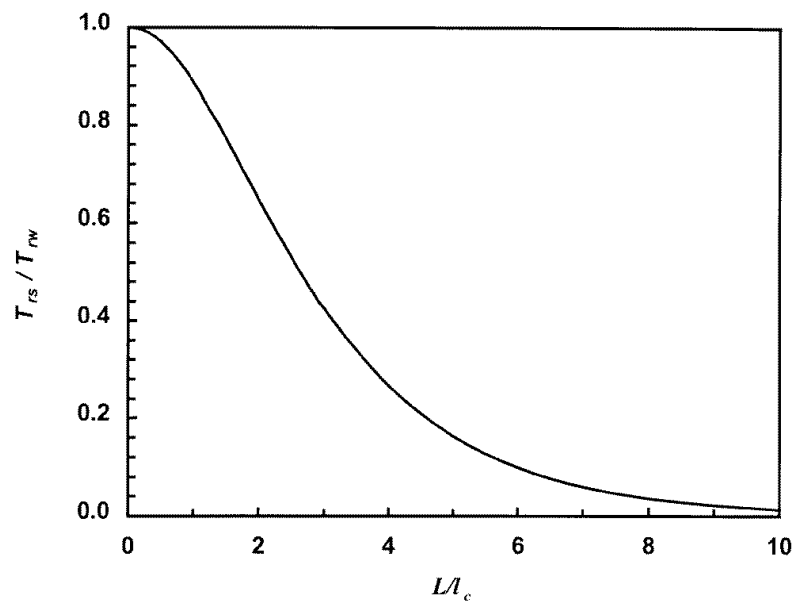

Figure 17. Dependence of sensor temperature on sensor properties.
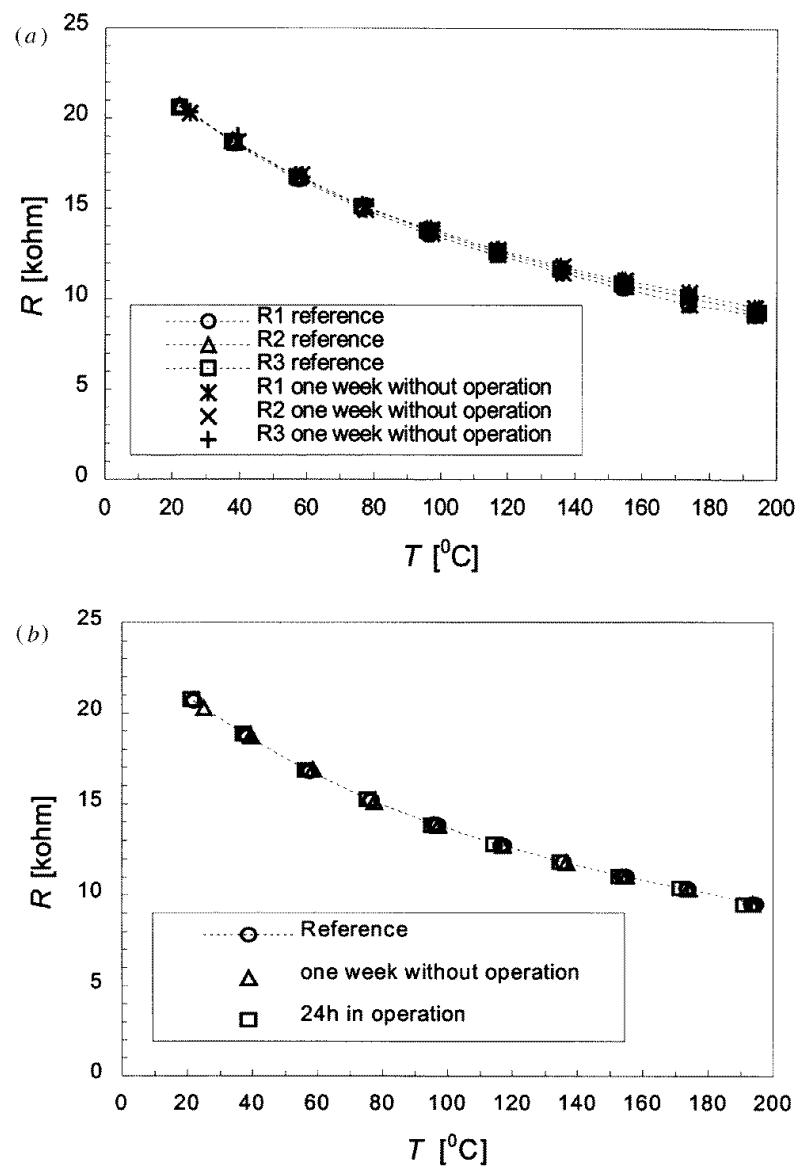

Figure 18. Long-term stability of sensor operation after $24 \mathrm{~h}$ : (a) without operation, and (b) in operation (SiGe, $4 \times 4 \mu \mathrm{m}^{2}$, phosphorus doped, $\left.1.25 \times 10^{19} \mathrm{~cm}^{-3}, 50 \mu \mathrm{A}\right)$.

$1 \%$. Moreover, the difference between the sensor calibration curves before and after $24 \mathrm{~h}$ in operation (figure $18(b)$ ), is also less than $1 \%$. Hence, drift of less than 0.01 in the resistance of the bridge sensors with or without operation can be obtained after an appropriate burn-in process.

\section{Conclusions}

Bridge-like microthermistors based on three polycrystalline films: $\mathrm{Si}, \mathrm{SiGe}$ and $\mathrm{Ge}$, have been fabricated and characterized. The poly-Si data are consistent with previous results while the poly-SiGe and poly-Ge data are available for the first time. So far there is no indication that the performance of one of the materials is clearly better than the others. An air gap between the thermistor and substrate decreases the thermal mass, but the resulting improvement of the frequency response is not very significant. The temperature distribution along the bridge due to current heating can be modelled very well. At steady-state conditions, by far the dominant heat transfer mechanism is conduction, while radiation can be neglected altogether. Heat convection is relatively small and thus the bridge temperature is not very sensitive to the ambient gas velocity or Reynolds number. The bridge temperature depends on three groups of parameters: material properties, geometrical dimensions and operating conditions. A characteristic length scale, resulting from the steady-state energy balance, controls the sensitivity of the bridge. The smaller is the characteristic length scale, the higher is the bridge sensitivity. The long-term stability of a SiGe bridges has been studied. The repeatability after an extended period of time with or without operation is good, less than $1 \%$ difference, due to the burn-in process.

\section{Acknowledgments}

This work was sponsored by RGC grant HKUST579/94E of the Hong Kong Research Grants Council. The authors would like to thank Mr Wan Lap Yeung, Mr Zhonghe Jin and Mr Miton Yeung for their assistance in the fabrication and the experimental set-up.

\section{References}

Blackwelder R F 1981 Hot-wire and hot-film anemometers Methods of Experimental Physics: Fluid Dynamics vol 18, ed R J Emrich (New York: Academic) part A

Churchill S W and Bernstein M 1977 A correlating equation for forced convection from gases and liquids to a circular cylinder in crossflow J. Heat Transfer Trans. ASME 94 300-6

Gardner J W 1994 Microsensors: Principles and Applications (Chichester: Wiley)

Ho C M, Tung S, Lee J B, Tai Y C, Jiang F and Tsao T 1997 MEMS - A technology for advancements in aerospace engineering AIAA Paper 97-0545

Jiang F PhD Thesis 1997 California Institute of Technology, USA

Jiang F, Tai Y C, Ho C M and Li W J 1994 A micromachined polysilicon hot-wire anemometer Solid-State Sensor and Actuator Workshop June 13-16

Jiang L, Wang Y, Wong M and Zohar Y 1998 Microchannels with suspended temperature sensors for heat transfer study Fluidics '98, Applications of Micro-Fabrication to Fluid Mechanics 1998 International Mechanical Engineering Congress and Exposition (Anaheim, CA, November 15-20) accpeted

Jorsh G J and Muller R S 1978 Conduction properties of lightly doped polycrystalline silicon Solid State Electron. 211045

Lu N C C, Gerzberg and Meindl J D 1978 A quantitative model of the effect of grain size on the resistivity of polycrystalline silicon resistors IEEE Electron Device Lett. 138

Mastrangelo C $1990 P h D$ Thesis University of California at Berkeley, CA 
L Jiang et al

Obermeier E, Kopystynski P and Nieble R 1986 Characteristics of polysilicon layers and their application in sensors IEEE Solid-State Sensors Workshop

Tai Y C, Mastrangelo C H and Muller R S 1988 Thermal conductivity of LPCVD polycrystalline silicone $J$. Appl. Phys. 63 1442-7

Tai Y C and Muller R S 1985 Polysilicon bridge for anemometer application Digest Tech Paper, Transducers '85 (Philadelphia, PA, June 4-7) pp 354-7

1988 Lightly doped polysilicon bridge as a flow meter Sensors Actuators 15 63-75

Wood M H, Muzik A, Huston H H and Hazara R 1993 Burn-in Microelectronics Manufacturing Diagnostics Handbook ed A H Landzberg (New York: Van Nostrand Reinhold) 